\title{
CONQUEST: A Quality Improvement Program for Defining and Optimizing Standards of Care for Modifiable High-Risk COPD Patients
}

\author{
Luis Alves $\mathbb{D}^{1}$, Rachel Pullen $\mathbb{D}^{2}$, John R Hurst $\mathbb{D}^{3}$, Marc Miravitlles $\mathbb{D}^{4}$, Victoria Carter ${ }^{5}$, \\ Rongchang Chen ${ }^{6}$, Amy Couper (D) ${ }^{2}$, Mark Dransfield ${ }^{7}$, Alexander Evans ${ }^{5}$, Antony Hardjojo (D) ${ }^{2}$, \\ David Jones $\mathbb{D}^{5}$, Rupert Jones $\mathbb{D}^{8}$, Margee Kerr ${ }^{5}$, Konstantinos Kostikas $\mathbb{D}^{9}$, Jonathan Marshall ${ }^{10}$, \\ Fernando Martinez (iD ${ }^{11}$, Marije van Melle $\mathbb{D}^{5}$, Ruth Murray ${ }^{5}$, Shigeo Muro $\mathbb{D}^{12}$, Clementine Nordon ${ }^{10}$, \\ Michael Pollack ${ }^{13}$, Chris Price ${ }^{5}$, Anita Sharma ${ }^{14}$, Dave Singh ${ }^{15}$, Tonya Winders ${ }^{16}$, David B Price (iD ${ }^{2}$
}

\begin{abstract}
'EPIUnit - Epidemiology Research Unit, ITR - Laboratory for Integrative and Translational Research in Population Health, Instituto de Saúde Pública da Universidade do Porto, Porto, Portugal; ${ }^{2}$ Observational and Pragmatic Research Institute, Singapore, Singapore; ${ }^{3}$ UCL Respiratory, University College London, London, UK; ${ }^{4}$ Pneumology Dept, Hospital Universitari Vall d'Hebron, Vall d'Hebron Institut de Recerca (VHIR), Vall d'Hebron Barcelona Hospital Campus, CIBER de Enfermedades Respiratorias (CIBERES), Barcelona, Spain; ${ }^{5}$ Optimum Patient Care, Cambridge, UK; ${ }^{6}$ Key Laboratory of Respiratory Disease of Shenzhen, Shenzhen Institute of Respiratory Disease, Shenzhen People's Hospital (Second Affiliated Hospital of Jinan University, First Affiliated Hospital of South University of Science and Technology of China) Shenzhen, Guangdong, People's Republic of China; ${ }^{7}$ Division of Pulmonary, Allergy, and Critical Care Medicine, Lung Health Center, University of Alabama at Birmingham, Birmingham, AL, USA; ${ }^{8}$ Research and Knowledge Exchange, Plymouth Marjon University, Plymouth, UK; ${ }^{9}$ Respiratory Medicine Department, University of loannina School of Medicine, loannina, Greece; ${ }^{10}$ BioPharmaceuticals Medical, AstraZeneca, Cambridge, UK; ${ }^{11}$ New York-Presbyterian Weill Cornell Medical Center, New York, NY, USA; ${ }^{12}$ Department of Respiratory Medicine, Nara Medical University, Nara, Japan; ${ }^{13}$ BioPharmaceuticals Medical, AstraZeneca, Wilmington, DE, USA; ${ }^{14}$ Platinum Medical Centre, Chermside, QLD, Australia; ${ }^{15}$ Division of Infection, Immunity \& Respiratory Medicine, University of Manchester, Manchester, UK; ${ }^{16}$ USA \& Global Allergy \& Airways Patient Platform, Vienna, Austria
\end{abstract}

Correspondence: David B Price, Observational and Pragmatic Research Institute, 22 Sin Ming Lane, \#06-76, Midview City, 573969, Singapore, Tel +65 3105 I489, Email dprice@opri.sg

Abstract: The COllaboration on QUality improvement initiative for achieving Excellence in STandards of COPD care (CONQUEST) aims to improve the quality of COPD care in primary care. The CONQUEST target population includes patients diagnosed with COPD, and those undiagnosed but with COPD-like exacerbations, who are at high but modifiable risk of COPD exacerbations, increased morbidity, and mortality. Timely diagnosis and optimized management to reduce these risks is vital. There is a need for a quality improvement program (QIP) that enables long-term improvement of patient clinical outcomes via integration of the program into routine clinical care. Core to the CONQUEST program is the adoption of four specifically designed, globally applicable, and expert-agreed quality standards (QS) for modifiable high-risk COPD patients. Translation of the QS into clinical practice, and implementation of the QIP, is guided by the CONQUEST global operational protocol provided to sites meeting the minimum healthcare system requirements. Initial analyses of current practices are conducted to generate baseline assessments of need within healthcare systems and sites looking to implement the QIP. Implementation is supported by the provision of CONQUEST resources and tools, such as clinical decision support, that promote prompt identification and treatment of patients. Utilization of electronic medical record (EMR) and patient-reported data are integral components of the QIP. Regular, automated collection and analysis of data, combined with a cyclical review of the implementation process, will be conducted for long-term, continuous improvement and health impact evaluation. The CONQUEST QIP will be an important resource in the identification and management of patients with modifiable high-risk COPD. Embedding the CONQUEST QS into routine clinical practice with regular evaluation and feedback will result in long-term quality of care improvement.

Keywords: patient-reported outcome, patient-reported information, clinical decision support, primary care, quality standards, integrated care

Received: 26 October 2021 Accepted: 2 February 2022 Published: 23 February 2022 


\section{Introduction}

\section{The Need to Optimize COPD Diagnosis and Management}

Chronic obstructive pulmonary disease (COPD) is a chronic lung disease characterized by a progressive deterioration in lung function and airflow limitation. ${ }^{1}$ Despite opportunities for prevention and treatment, COPD remains one of the leading causes of morbidity and death globally, contributing to an estimated 3.23 million deaths in 2019. ${ }^{1,2}$ COPD increases the risk of developing other chronic respiratory and cardiovascular conditions, and imposes substantial socioeconomic impact and reduction in quality of life. ${ }^{1-3-5}$

Early identification of COPD is critical for prompt initiation of lifestyle changes and therapy. ${ }^{6}$ Delays can contribute to worse disease outcomes including a higher risk of exacerbations (episodes of acute worsening of respiratory symptoms). ${ }^{7,8} \mathrm{COPD}$ exacerbations are associated with increased cardiovascular risk, ${ }^{9,10}$ faster lung function decline, ${ }^{11,12}$ reduced health-related quality of life, ${ }^{13}$ and increased mortality. ${ }^{14,15}$ Yet many COPD patients do not receive timely diagnosis, with as many as $85 \%$ having recorded consultations for lower respiratory symptoms in the 5 years prior to diagnosis. ${ }^{16}$ Even within those diagnosed with COPD, we observed that patients often do not receive initiation with the appropriate therapy, nor have their treatment adjusted accordingly as the disease progresses. ${ }^{6}$ In addition, patients with more severe symptoms and frequent exacerbations appeared to be undertreated. ${ }^{6}$ There is therefore a need for a strategy to enable earlier identification and to optimize the management of symptomatic COPD.

\section{Quality Improvement Programs in COPD}

Quality improvement programs (QIPs) are a set of activities to systematically analyze and review current standards and practices of care to identify areas and strategies for improvement, implement change, and analyze the impact of that change. ${ }^{17,18}$ Successful QIPs follow a logical, iterative process with time for reflection and analysis of program impact that promotes beneficial modifications in routine clinical approaches.

Previous QIPs in COPD include initiatives that have focused on early identification of COPD, reducing hospital re-admissions for exacerbations, encouraging multi-disciplinary approaches to care, educational tools, self-management plans, and guideline adherence. ${ }^{19-26}$ Programs focused on integrated care and disease management have shown beneficial patient outcomes, reporting improvements in patient quality of life and hospitalization rates for exacerbations. ${ }^{26-29}$ However, overall results of previous QIPs on COPD exacerbations of different severities have been mixed, and a long-term reduction of exacerbations has not been consistently demonstrated. ${ }^{30-33}$ This may have been due to the short-term nature of some of the initiatives, which did not involve long-term monitoring or evaluation of improvement in patient clinical outcomes. ${ }^{34,35}$ Heterogeneity in clinical trial design and outcome reporting for COPD QIPs suggests a lack of standardized outcome sets for outcomes such as exacerbations, and this may contribute to the mixed picture observed. ${ }^{30}$ Additionally, there is a lack of utilization of patient-reported outcomes (PRO) and patient-reported information (PRI) data which may be important to guide quality improvement by providing additional information on the patients' clinical status. ${ }^{28,36,37}$ Results from previous work therefore support the need for a QIP that includes patientreported data, is integrated into routine care, and that evaluates exacerbation rates and other patient outcomes over a longer time frame.

\section{Background and Aims of the CONQUEST Quality Improvement Program}

Optimum Patient Care (OPC), as a non-profit social enterprise, has provided sustainable QIPs to healthcare professionals across several countries. ${ }^{38-40}$ A previous QIP in asthma led by OPC has successfully reduced the rate of asthma exacerbation from $24.8 \%$ pre-implementation to $15.3 \%$ in the year post-implementation, while no improvement was observed among control sites. ${ }^{40}$ OPC also has a well-established track record in COPD research in primary care ${ }^{41-44}$ including the establishment of a COPD patient registry. ${ }^{45,46} \mathrm{~A}$ recent evaluation of a COPD QIP led by OPC demonstrated an overall $20 \%$ reduction of COPD exacerbations, compared to $10 \%$ in control practices, in the year following implementation. ${ }^{38}$

In July 2020, OPC Global, in a collaborative effort with AstraZeneca and the Observational and Pragmatic Research Institute (OPRI), established the COllaboratioN on QUality improvement initiative for achieving Excellence in STandards of COPD care (CONQUEST) program as the first-of-its-kind QIP with data which builds into a collaborative and interventional COPD registry (https://conquest.care/). ${ }^{47}$ The program involves multi-level stakeholder engagement 
including clinicians, researchers, healthcare professionals, patients, and a panel of international experts forming the CONQUEST Global Steering Committee. The CONQUEST data source is registered with the European Network of Centres for Pharmacoepidemiology and Pharmacovigilance (ENCEPP/DSPP/42512).

The CONQUEST QIP was conceived with a vision to drive long-term positive change in the management of patients with modifiable high-risk COPD. Such patients are considered to have a higher risk of adverse health outcomes, but that risk may be modifiable through optimized disease management (further elaborated in the CONQUEST target population section below). While the focus of CONQUEST QIP is on the population considered to have modifiable high-risk COPD, the program also supports the adoption of guideline-led clinical decision-making for all COPD patients.

The CONQUEST QIP aims to address some of the challenges and barriers of implementing quality improvement initiatives in primary care, including difficulties in keeping up with current guidelines, limited availability of guidelines in an accessible format at the point of care, and difficulty in analyzing current practice to identify areas where change is required. ${ }^{38}$ These barriers, and the solutions taken by OPC to mitigate them, are illustrated in Figure 1. Mindful of these issues, CONQUEST takes a cyclical quality improvement approach, including embedding the program within healthcare systems, and integrating electronic medical records (EMR) with PRO and PRI data at all stages of the QIP - from patient identification to data analysis. Clinical decision support (CDS) and regular feedback mechanisms are utilized to facilitate earlier, targeted, and risk-based assessment and management. Coordinated and integrated care in quality improvement, combined with ongoing learning and use of technology, have been utilized in a number of QIPs for chronic disease across Europe ${ }^{48,49}$ and are methods supported by professional quality improvement organizations. ${ }^{50,51}$ Furthermore, the patient-centric approach in CONQUEST is congruent with a recently published, globally-applicable, COPD patient charter $^{52}$ to proactively identify and prevent exacerbations.

This article outlines the key features and requirements of the CONQUEST QIP, as illustrated in Figure 2. We describe the CONQUEST Quality Standards (QS), the definition of the modifiable high-risk target population, the global operational protocol, and the minimum healthcare system requirements for QIP implementation. This article will also discuss the activities involved in the implementation of the QIP into routine clinical practice, including the use of PRO measures and PRI, and regular data collection and analysis to gauge the outcome of QIP implementation. Lastly, the steps to be taken to evaluate the impact of program implementation within participating sites are also described.

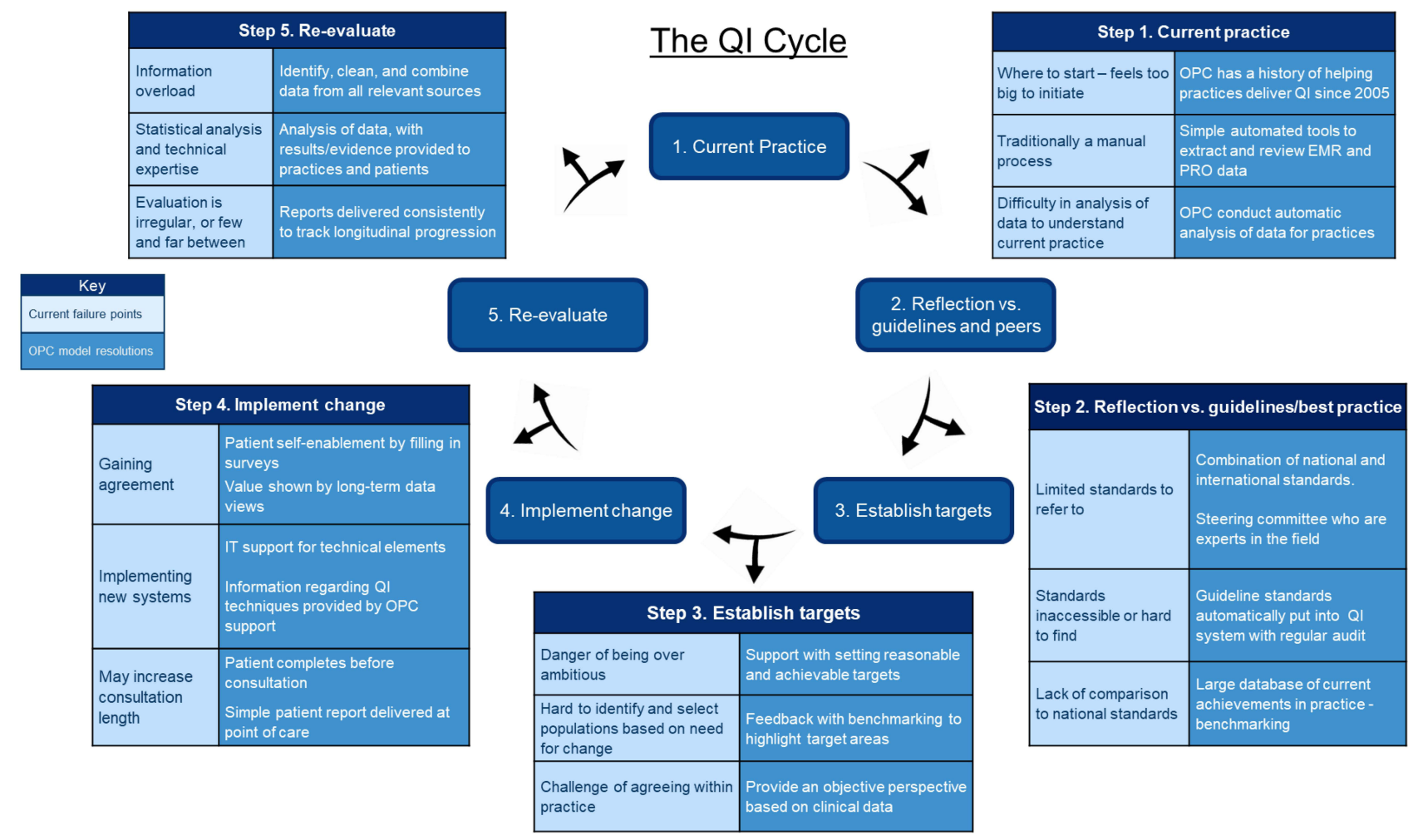

Figure I The cycle of quality improvement in primary care: Barriers and OPC solutions. Adapted from Evans et al. ${ }^{38}$

Abbreviations: EMR, electronic medical records; IT, information technology; OPC, optimum patient care; PRO, patient-reported outcomes; QI, quality improvement. 


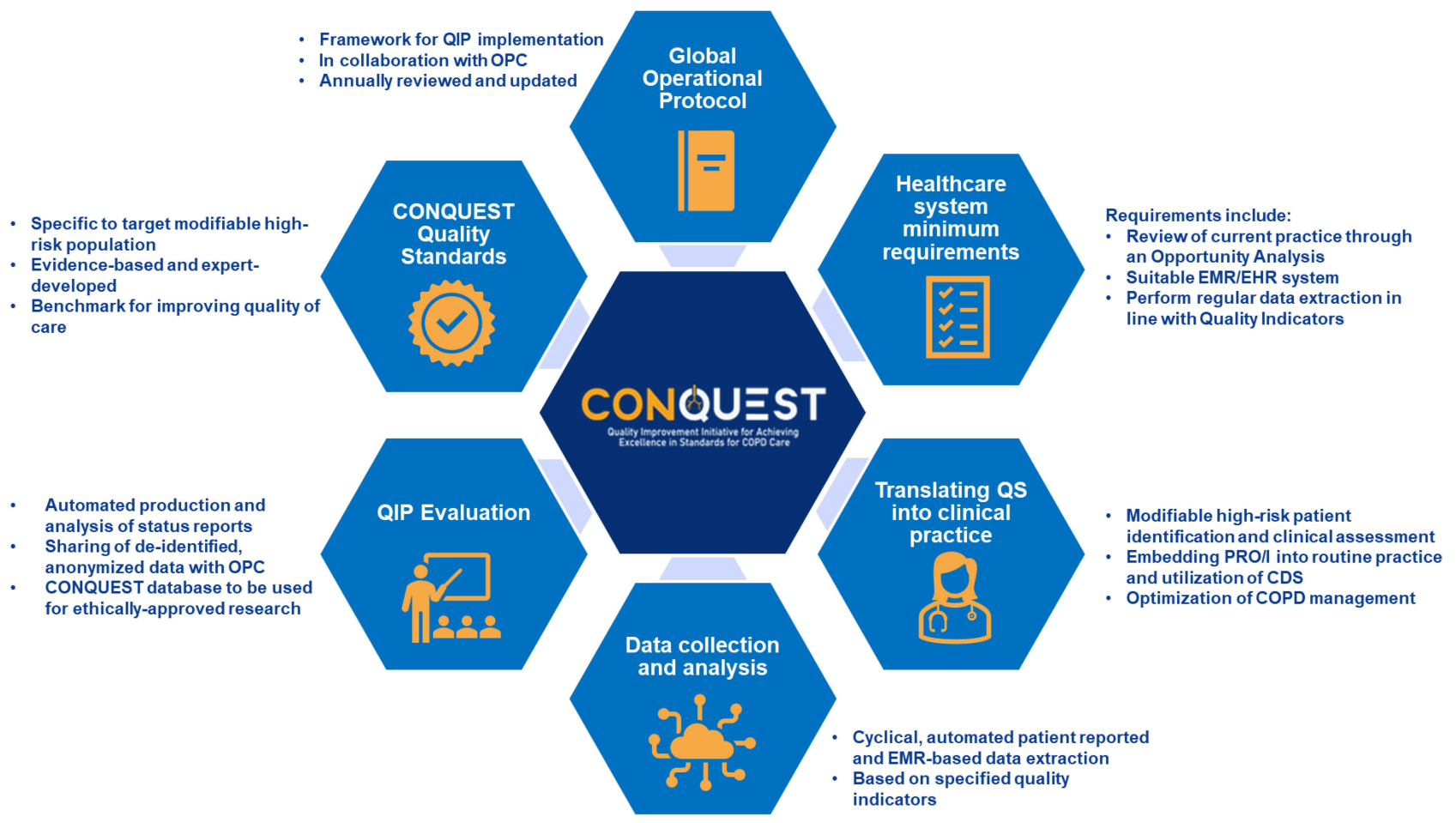

Figure 2 Key features and requirements of the CONQUEST QIP.

Abbreviations: CDS: Clinical Decision Support; CONQUEST: COllaboratioN on QUality improvement initiative for achieving Excellence in STandards of COPD care; COPD: Chronic Obstructive Pulmonary Disease; EMR/EHR: Electronical medical records/electronic health records; PRI: Patient-Reported Information; PRO: Patient-Reported Outcomes; QIP: Quality Improvement Program; OPC: Optimum Patient Care; QS: Quality Standards.

\section{The CONQUEST Quality Improvement Program The CONQUEST Quality Standards}

At the heart of the CONQUEST program is the development of the QS and their translation into the operational protocol. The CONQUEST QS are evidence-based, globally applicable standards that represent a roadmap by which participating sites can improve the quality of care and health outcomes for patients with modifiable but high-risk COPD. The QS will also serve as a benchmark to monitor the impact of quality improvement in COPD management for this population. ${ }^{38}$

The CONQUEST QS are founded upon the consolidation of national and international COPD quality standards, guidelines and strategies, and a review of recent relevant COPD research. ${ }^{47}$ The standards were developed through consensus from a panel of 11 internationally recognized experts in COPD research who form the CONQUEST global steering committee. The global steering committee agreed upon a final set of quality standards that cover (Figure 3): 1) identification of the target population (QS1), 2) assessment of disease and quantification of future risk (QS2), 3) nonpharmacological and pharmacological intervention (QS3), and appropriate follow-up (QS4).

The rationale behind each quality standard and its supporting evidence have been comprehensively described in a separate publication. ${ }^{47}$ The activities and tools used to translate the standards into clinical practice are described in the "translation of quality standards into clinical practice" section below.

\section{The CONQUEST Patient Population}

The "modifiable high-risk" patient population to whom the CONQUEST QS will be applied includes those already diagnosed with COPD, and those who do not have a COPD diagnosis but have a history of smoking and COPD-like exacerbations (subsequently referred to as exacerbations of potential COPD) indicating that a COPD diagnosis is likely. Exacerbations are defined as significant worsening of respiratory symptoms which can be either moderate (requiring an 


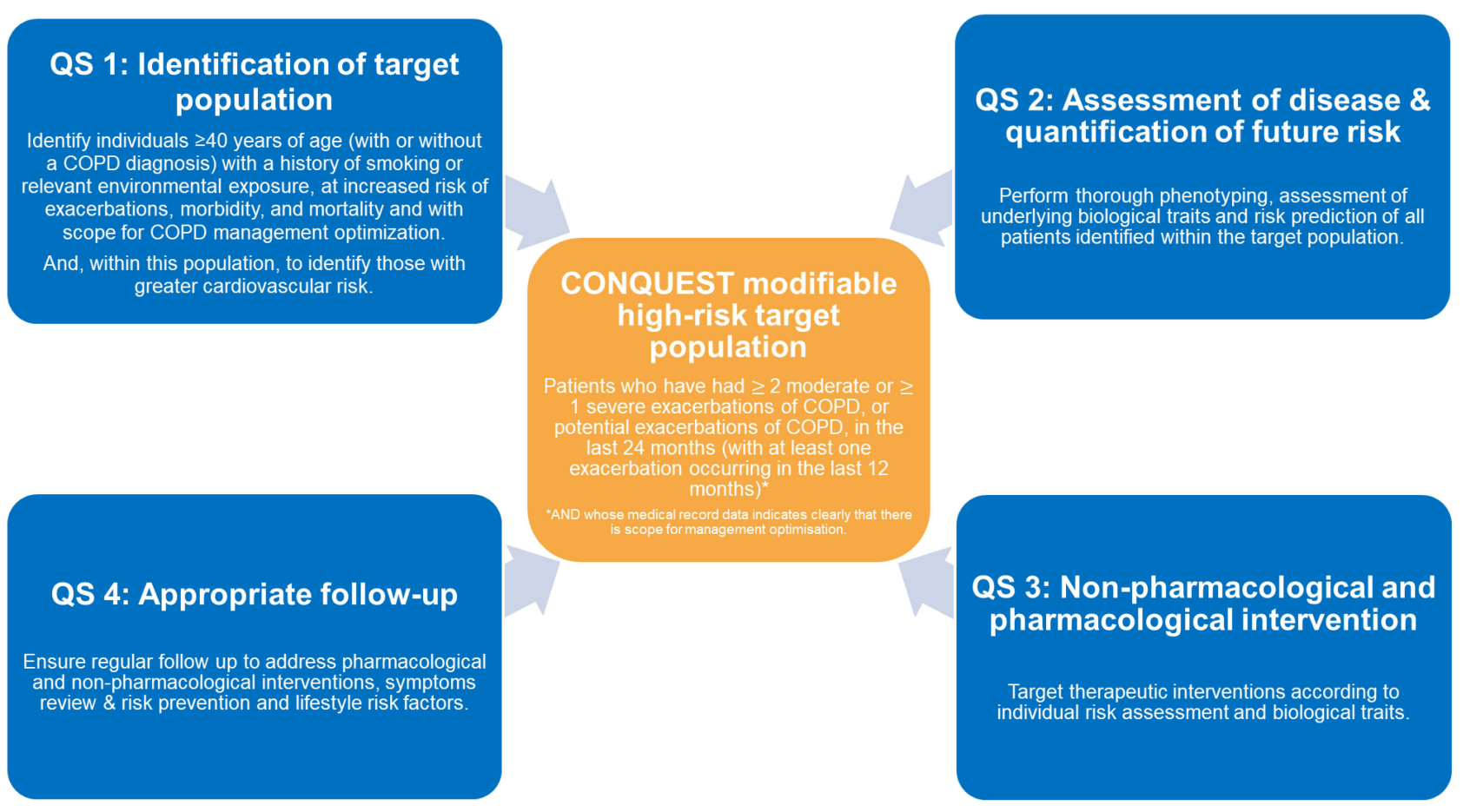

Figure 3 The CONQUEST Quality Standards and target population.

Abbreviations: CONQUEST: COllaboratioN on QUality improvement initiative for achieving Excellence in STandards of COPD care; COPD: Chronic Obstructive Pulmonary Disease; QS: Quality Standards.

acute course of systemic corticosteroid and/or antibiotics, or emergency room visit) or severe (resulting in hospital admission). Modifiable high-risk patients are defined as patients who have had 2 or more moderate, or 1 or more severe exacerbations of COPD or potential COPD in the last 24 months, with at least one exacerbation occurring in the last 12 months, and whose medical record data indicates clearly that there is scope for management optimization. Scope for optimization includes patients who continue to have exacerbations whilst on their current therapy (indicating a need for therapy review) and/or whose management may be optimized by correct diagnosis, modification of prescribed therapy, or non-pharmacological interventions, such as smoking cessation interventions, active lifestyle encouragement, or pulmonary rehabilitation.

Early identification of opportunities to improve management for these modifiable high-risk patients will result in decreased frequency of exacerbations, better symptom control, and decreased risk of lung function decline. The strong link between COPD exacerbations and the risk of major adverse cardiovascular events ${ }^{9,53}$ further highlights the importance of proactively identifying the modifiable high-risk patients. Lastly, targeting this population of patients will present opportunities for clinicians to assess and treat other co-morbidities, and recommend vaccination for influenza, pneumococcal infection, and COVID-19.

\section{CONQUEST Global Operational Protocol}

A global operational protocol will be provided to sites looking to implement the CONQUEST QIP who meet the minimum requirements for implementation discussed in the following section on healthcare system or site 'minimum requirements'. The protocol describes the core framework of the QIP, and steps for implementation within regional and national healthcare systems. CONQUEST recognizes that there are regional, national, and international differences in healthcare systems and context, therefore suitable alternatives for certain program components are provided within the protocol where appropriate, and where their use does not undermine or impact upon the core program requirements. The global operational protocol will be annually reviewed, taking into account information and feedback from participating 
sites, members of the global steering committee, and emerging evidence in the field to ensure ongoing relevance to primary care. The current version is available from https://conquest.care/.

\section{Healthcare System or Site "Minimum Requirements" for Implementing and Delivering CONQUEST}

The implementation of the QS and core QIP elements into a healthcare system or practice comes with a minimum set of requirements. A checklist has been developed to enable potential healthcare sites to determine the feasibility of the program in their locality (Table 1). The CONQUEST QIP is designed to be flexible enough to accommodate the impact of local context, for example, COVID-19 restrictions. However, participating sites are mandated to ensure the minimum CONQUEST standards are fulfilled. Any healthcare system or site implementing the CONQUEST program should do so in collaboration with OPC and, wherever possible (subject to local legal and ethical requirements), share de-identified data with OPC to facilitate ongoing program evaluation, benchmarking with other sites, and future collaborative research.

The infrastructure, resources, clinical activities, data handling, and information sharing requirements that form the basis of the minimum requirement checklist to implement CONQUEST are described in Table 1. Infrastructure and resources described include the need for a local implementation team, an EMR system capable of supporting the program, and access to the equipment and expertise necessary for disease identification and management such as spirometry. Options for alternatives to traditional spirometry may be considered given restrictions imposed by the COVID-19 pandemic (such as measures of peak flow or remote spirometry). Importantly, the need for sites to review their current practice and outcomes in the form of an "opportunity analysis" is a core requirement of the program. This involves retrospective EMR data analysis of COPD patients within a healthcare system to identify areas where opportunities for management optimization exist.

Clinical activity requirements outlined in Table 1 are to be considered in tandem with the clinical activities described in Figure 4, and sites should consider what barriers might occur in implementation. In particular, CONQUEST advocates piloting EMR-based search algorithms related to patient identification to ensure local terms used will result in similar cohorts to the intended CONQUEST populations. Countries may also wish to consider how they will operationalize this aspect if their system relies more heavily on free text than diagnostic codes. National guidelines may be used in place of the Global Initiative for Chronic Obstructive Lung Disease (GOLD) recommendations where these do not undermine the program core components. Again, local context may require alternative strategies to some clinical activities such as annual spirometry.

The CONQUEST QIP aims to create an inclusive and collaborative network centered around improving COPD care and management. Data-related requirements outlined in the checklist, including data analysis and information sharing, are therefore necessary to create a global network that takes into consideration the ethical and privacy requirements in each country. A data-sharing environment creates opportunities to build diverse and globally representative datasets, encourage learning and ongoing improvement, and create a network of people implementing CONQUEST internationally.

\section{Implementation of the CONQUEST QIP}

Figure 4 illustrates the key elements of the implementation of CONQUEST QIP into routine clinical practice. OPC will support QIP implementation through the provision of the global operational protocol and the resources it contains, which include templates for questionnaires and patient appointment invitation letters. OPC will also provide technical expertise on patient identification algorithms and methods that can be implemented within the sites to automate the collection and analysis of EMR, PRO, and PRI data. ${ }^{38}$

Participating sites will also receive access to the CONQUEST CDS specifications, which may be appropriately tailored to local or national circumstances. These include clinical considerations for healthcare professionals and site-led education and training activities. CDS tools may prompt healthcare professionals to consider COPD diagnostic assessment, the need for further COPD status assessments, pharmacological and non-pharmacological management 
Table I Minimum requirements checklist for site implementation of CONQUEST QIP

Site Requirements for Program Implementation
Infrastructure and resources
Build an Implementation team consisting of representatives from primary and secondary care, administrative, managerial, and
clinical staff with necessary payer-provider level support.
Have a Practice or healthcare system EMR/EHR capable of supporting patient identification using CONQUEST search
criteria and data extraction of de-identified patient data for analysis (with appropriate ethical approvals in place).
Agree to pilot EMR data extraction using site systems to ensure concordance with expected populations and review of current
practice in the form of an Opportunity Analysis.
Have the administrative ability and system capacity to contact patients and schedule assessment appointments for identified
patients within 3 months of identification (eg, via mail, phone, text etc.).

Access to spirometry - equipment at practice with trained staff or prompt referral route for service provision (COVID-19 impact to be considered and alternatives such as remote spirometry may be acceptable).

Utilization of Clinical Decision Support (CDS) algorithms for healthcare practitioners

Perform annual Modifiable high-risk patient population identification using EMR-based search criteria provided by OPC in $\quad \square$ the Global Operational Protocol.

Perform diagnostic assessment within 3 months for patients identified as "undiagnosed with potential modifiable high-risk COPD", to include measurement of lung function (spirometry) and clinical correlation of symptoms.

Perform a disease and risk assessment for all modified high-risk COPD patients, including newly-diagnosed COPD patients, within 3 months in accordance with CONQUEST operational protocol.

Ensure at least annual distribution (and data collection) of PRO/PRI Questionnaires to COPD patients.

Initiate non-pharmacological interventions for modifiable high-risk patients (smoking cessation, pulmonary rehabilitation etc.) in accordance with disease assessment findings.

Prescribe initial and maintenance therapy in accordance with current GOLD recommendations (or national guidelines), aided by CDS algorithms.

Apply COPD control criteria at clinical review to guide management and repeat every 3 months (with a clinical patient review) after a change in therapy, or until COPD control achieved.

Ensure all patients identified as modifiable high-risk (both already diagnosed and newly diagnosed) have clinical review and follow up 3-monthly until detailed review suggests no further management optimization interventions are appropriate; then at least annual patient follow-up.

Data analysis

Perform at least annual data extraction and analysis of PRO/PRI data and de-identified EMR data in line with CONQUEST Quality Indicators.

Present data extracted in relation to Quality Indicators in practice, health system or physician level status reports as appropriate to local context (may be performed by suitable third party).

Information and data sharing

Share healthcare system or practice status reports with healthcare practitioner and implementation teams for continuous refinement of the program, comparison of current practice against the CONQUEST Quality Standards and identification of areas for ongoing improvement. 
Table I (Continued).

\begin{tabular}{|l|l|}
\hline Site Requirements for Program Implementation & $\begin{array}{l}\text { Criteria } \\
\text { Met }\end{array}$ \\
\hline Make anonymized, de-identified EMR data available to OPC for benchmarking and use in ethically approved research & $\square$ \\
\hline Provide feedback on CONQUEST implementation and processes to OPC via website/annual meeting of CONQUEST sites. & $\square$ \\
\hline
\end{tabular}

Abbreviations: CDS, clinical decision support; CONQUEST, COllaboratioN on QUality improvement initiative for achieving Excellence in STandards of COPD care; COPD, chronic obstructive pulmonary disease; EHR, electronic health records; EMR, electronic medical records; OPC, Optimum Patient Care; PRI, patient-reported information; PRO, patient-reported outcomes.

interventions, and timely patient review. The CONQUEST CDS is designed for the CONQUEST program based on national and international guidelines as well as expert insight to assist healthcare professionals in clinical decision making using EMR, PRO, and PRI data, and information obtained during consultations.

\section{Translation of Quality Standards into Clinical Practice}

The core activities in the implementation of the CONQUEST QIP encompass embedding the CONQUEST QS into routine care at participating healthcare sites and supporting healthcare professionals with PRO and PRI data collection, CDS tools, and regular data collection and analysis as illustrated in Figure 4.

Implementation of the QS begins with the identification of the CONQUEST target patient population, as described above, using validated EMR-based search algorithms ${ }^{35,43,54,55}$ adapted for CONQUEST (QS 1). Participating sites are responsible for the automated identification of patients meeting the modifiable high-risk criteria within their EMR system. Both patients who already have a COPD diagnosis and those currently undiagnosed with potential modifiable high-risk COPD will be identified. Those who do not have a COPD diagnosis are invited to a diagnostic case-finding appointment. If COPD is confirmed they, along with previously diagnosed patients, will go on to have a disease assessment (QS 2). Alternative diagnoses, such as asthma or cardiac disease, will be sought if current diagnostic assessment excludes COPD.

Implementation of QS2 involves scheduling disease assessment appointments for already-diagnosed and newlydiagnosed COPD patients meeting the modifiable high-risk criteria. At assessment, disease phenotyping and assessment of future risk of exacerbations and adverse health outcomes is performed via evaluation of underlying patient characteristics, clinical traits, and disease history. Core activities of QS 2 include health status assessment using the COPD Assessment Test (CAT), recording of exacerbation history, performing post-bronchodilator spirometry, measurement of blood eosinophil count, and cardiovascular risk assessment. Relevant investigations are performed and information is obtained through the automated, systematic collection of EMR, PRO, and PRI data to enable targeted phenotype-specific management to optimize treatment outcomes.

QS3 promotes guideline-appropriate treatment initiation and maintenance therapy adjustment according to the clinical information obtained from the disease assessment of QS2. This is supported by the use of CDS and the COPD control tool $^{56}$ (as described below in the PRO and PRI section) to achieve swift progress to optimum management. ${ }^{57}$ Nonpharmacological intervention and consideration of the need for cardiovascular therapy are also an important part of QS 3. CDS tools will assist clinical decision-making by providing healthcare professionals with relevant patient information, from previously collected EMR, PRO, and PRI data, and considerations for pharmacological therapy at every consultation.

Lastly, translating QS 4 into practice requires regular, ongoing review of COPD patients in line with the clinical need to modify disease pathways. Thus, ensuring the continuation of behavioral changes, and improvement in quality of care for COPD patients through regular patient consults, informed by feedback from the QIP. Core follow-up activities include symptom and medication review, a repeat of CAT and COPD control PRO measures, review of inhaler technique, and specialist referrals, including referral for pulmonary rehabilitation, as required. Other non-pharmacological measures such as provision of lifestyle advice and vaccinations are also encouraged. The frequency of scheduling of further review 


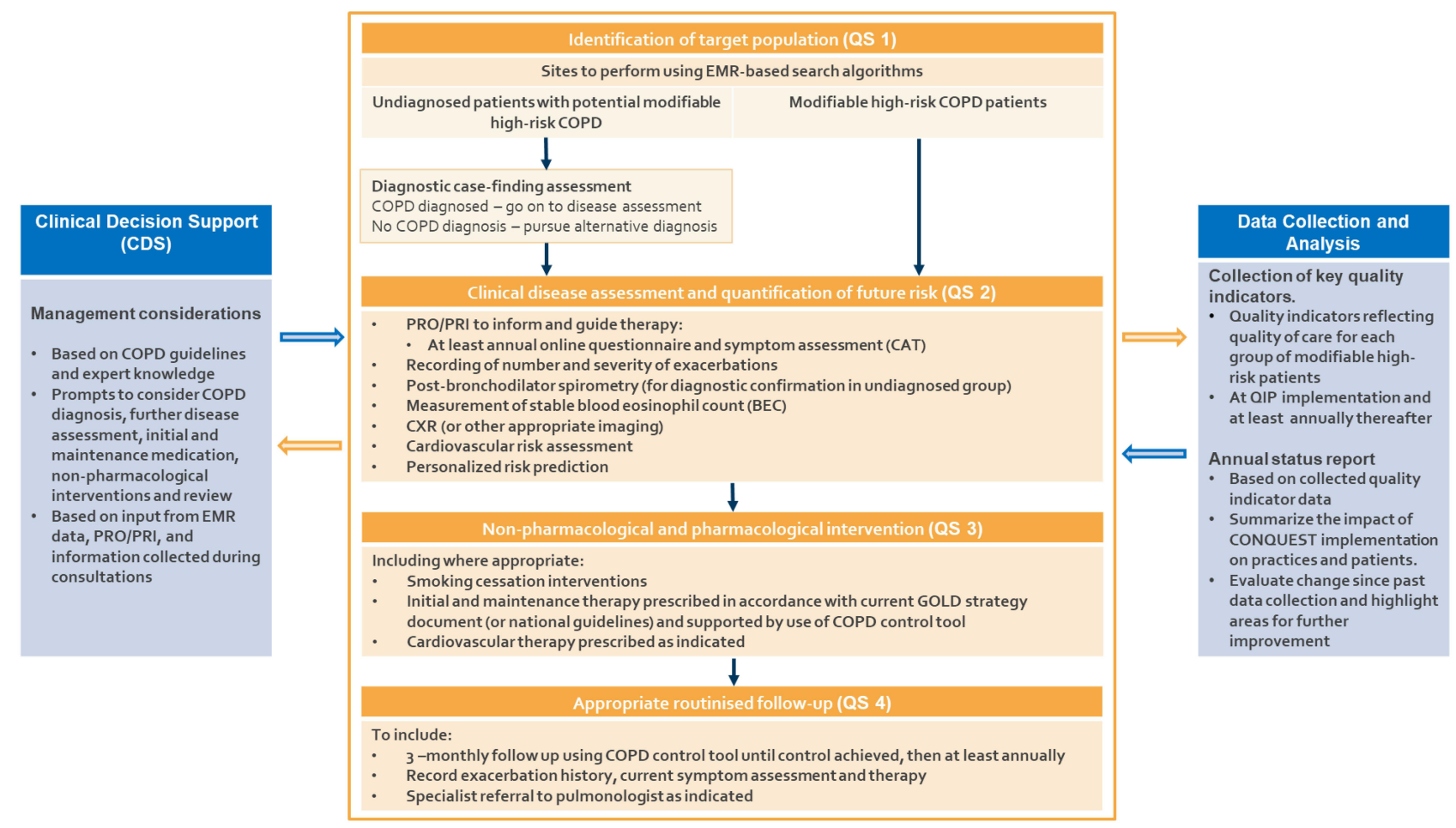

Figure 4 Key elements for translating CONQUEST Quality Standards into practice.

Abbreviations: BEC, blood eosinophil count; CDS, Clinical Decision Support; CONQUEST, COllaboratioN on QUality improvement initiative for achieving Excellence in STandards of COPD care; COPD, chronic obstructive pulmonary disease; CAT, Chronic Obstructive Pulmonary Disorder Assessment Test; CCQ, Clinical COPD Questionnaire; CXR, Chest X-Ray; EMR, Electronic Medical Record; GOLD, Global Initiative for Chronic Obstructive Lung Disease; mMRC, modified Medical Research Council; PRI, patient-reported information; PRO, patient-reported outcomes; QIP, quality improvement program; QS, quality standards.

is aided by the use of the COPD control tool. ${ }^{58}$ Follow-up should be scheduled at 3-monthly intervals until control is achieved; thereafter annual follow-up is required.

\section{Patient-Reported Outcomes (PRO) and Patient-Reported Information (PRI)}

CONQUEST supplements EMR data extraction reflecting diagnosis, care, and treatment provided with PRO and PRI data. PRO refer to health-related information that is obtained via validated tools and instruments completed by the patient, without clinician interpretation. ${ }^{59,60}$ PRI refers to additional information provided by patients in response to more general questions, such as how many cigarettes are smoked per day.

PRO measures are increasingly recognized as critical in the management of patients with COPD to obtain information beyond that which can be measured physiologically, such as spirometry, or obtained from EMR data. ${ }^{36,61}$ PRO provide valuable information that can be used to assess symptom burden and current disease status, guide therapy, and encourage patient involvement in managing their disease. From 2011, PRO assessed through the modified Medical Research Council (mMRC) Dyspnea scale and CAT have been incorporated into the GOLD recommendations as part of the ABCD assessment tool and thus inform recommendations for initial therapy. ${ }^{1}$

PRO and PRI collected as part of the CONQUEST QIP include a questionnaire to be completed at the time of, or within a month of, initial assessment and at least annually thereafter. Patients can access an online questionnaire with conditional functionality, ie, only relevant questions appear based on responses given. The questionnaire includes casefinding questions for those who do not have a COPD diagnosis, as well as questions on patient-reported symptom experience, exacerbation history, systemic steroid burden, inhaler use, smoking status, and treatment goals. Table 2 describes the general domains of the CONQUEST questionnaire and the justification for question inclusion.

CONQUEST also mandates participating practices to determine COPD control status, utilizing standardized questions, at every clinical COPD review the patient attends. ${ }^{58}$ COPD control incorporates the concept of the clinical impact of COPD reflecting the current clinical situation, and combines this with the stability of disease, comparing the patient's 
Table 2 CONQUEST Patient Questionnaire Domains and Instruments Utilized in Collection of Patient-Reported Outcomes and Information

\begin{tabular}{|c|c|c|}
\hline Questionnaire Domain & Instrument & Comments/Rationale for Inclusion \\
\hline Case-finding questions & $\begin{array}{l}\text { CAPTURE questionnaire }{ }^{63} \text { and } \\
\text { COPD diagnostic Questionnaire }\end{array}$ & $\begin{array}{l}\text { Only visible to those who do not have a COPD diagnosis } \\
\text { Aims to identify those with a greater likelihood of COPD }\end{array}$ \\
\hline $\begin{array}{l}\text { Health status: } \\
\text { a) Symptoms } \\
\text { (eg, breathlessness, cough, } \\
\text { sputum production and } \\
\text { appearance) } \\
\text { b) Physical/daily activities } \\
\text { c) Subjective perception }\end{array}$ & $\begin{array}{l}\text { a) mMRC Dyspnea scale, CAT, cough visual } \\
\text { analogue scale, COPD control tool } \\
\text { b) CAT, mMRC Dyspnea scale, COPD } \\
\text { control tool } \\
\text { c) COPD control tool }\end{array}$ & $\begin{array}{l}\text { Assessment of health status and disease burden } \\
\text { Helps guide therapy and management, eg, information } \\
\text { used in GOLD medication algorithms } \\
\text { Part of determining clinical impact and stability in the } \\
\text { COPD control tool }\end{array}$ \\
\hline $\begin{array}{l}\text { Exacerbations: } \\
\text { Frequency, severity, and } \\
\text { treatment }\end{array}$ & $\begin{array}{l}\text { CONQUEST Questionnaire and } \\
\text { COPD control tool }\end{array}$ & $\begin{array}{l}\text { Triangulation with EMR data - patient reporting of } \\
\text { exacerbation frequency may differ from EMR record. } \\
\text { Helps guide therapy and management decisions } \\
\text { Informs individual steroid burden. } \\
\text { Part of determining clinical stability in COPD control } \\
\text { tool and risk of future exacerbations }\end{array}$ \\
\hline $\begin{array}{l}\text { Inhaler use, technique and } \\
\text { adherence }\end{array}$ & $\begin{array}{l}\text { CONQUEST questionnaire and } \\
\text { COPD Control tool }\end{array}$ & $\begin{array}{l}\text { Essential to ensure correct use of therapy } \\
\text { Guides further management decisions } \\
\text { Part of determining clinical impact in COPD control too }\end{array}$ \\
\hline Smoking status & CONQUEST questionnaire & To prompt smoking cessation advice/intervention \\
\hline Goal setting and self-management & CONQUEST questionnaire & $\begin{array}{l}\text { Encourage patient engagement and self-empowerment } \\
\text { Information to correlate with EMR records, further detai } \\
\text { on steroid and antibiotic use. } \\
\text { Prompt creation or update of individual action plans } \\
\text { where appropriate }\end{array}$ \\
\hline
\end{tabular}

Abbreviations: CAPTURE, COPD Assessment in Primary Care to Identify Undiagnosed Respiratory Disease and Exacerbation Risk; CAT, COPD Assessment Test; CONQUEST, COllaboratioN on QUality improvement initiative for achieving Excellence in STandards of COPD care; COPD, chronic obstructive pulmonary disease; EMR, electronic medical record; GOLD, Global Initiative for Chronic Obstructive Lung Disease; mMRC, modified Medical Research Council.

current situation with previous visits. Control status provides information on the risk of future exacerbations for the individual, and the tool is therefore used within CONQUEST to prompt early therapy review for an uncontrolled patient. ${ }^{57}$

Collected and summarized PRO and PRI data are incorporated automatically into the EMR to achieve robust individual patient records that are correctly coded. The information obtained using these measures will be extracted along with EMR data to inform relevant quality indicators (described below), as well as providing valuable information for use in patient management.

\section{Data Collection and Analysis}

As part of the core QIP components, automated collection of key quality indicator data is performed before the implementation of the program, quarterly during the first year of implementation, and at least annually thereafter. Data collected must be anonymized and de-identified at the patient level. A delegated third party may be employed by the sites to conduct data collection and anonymization. The quality indicators are directly linked to the CONQUEST QS, reflecting key aspects of care for the modifiable high-risk target populations (including process outcome measures) and overall COPD care, and will be available to sites operating under the CONQUEST label. Participating sites are responsible for ensuring that ethical and data governance approvals are in place before initiating data collection. 
As noted in the QIP minimum requirements section, CONQUEST advocates initial data analysis and review of a site's current practice before implementation of the QIP. This initial review is crucial to highlight opportunities for further improvement.

The CONQUEST global operational protocol lists the quality indicators to be collected for each group of patients: undiagnosed patients with potential modifiable high-risk COPD, newly-diagnosed patients with modifiable high-risk COPD, and already-diagnosed patients with modifiable high-risk COPD. Briefly, the key quality indicators for undiagnosed patients include the percentage receiving a diagnostic assessment, treatment according to the diagnosis, and advice on smoking cessation. Quality indicators for COPD diagnosed patients cover parameters such as the percentage of patients receiving symptom and disease assessments, appropriate therapy and intervention, and follow-up review. Importantly, indicators also cover health outcomes such as the number of exacerbations experienced, changes in symptom burden, and rates of major events such as hospital admission for pneumonia or cardiac events.

\section{Evaluating the CONQUEST Quality Improvement Program Monitoring progress and sustain improvement}

The success of a QIP depends on embedding long-term positive change into the healthcare system. ${ }^{17,18}$ Therefore, one crucial component to achieving optimal standards of care is the cyclical review of the implementation process and outcomes achieved throughout the CONQUEST program. This review is vital to identify barriers towards implementation, and areas of further improvement, and also to maintain motivation towards change.

Quality indicator data collected will be analyzed to produce annual status reports. This process will be performed automatically within each site. Status reports will be used for benchmarking within individual sites and against other participating sites. The reports summarize the impact and success of the CONQUEST QIP for sites since the last data extraction point and highlight further areas for improvement. The status reports will include significant patient outcome measures such as symptom assessment scores, frequency of exacerbations, or other respiratory or cardiac events, hospitalizations, and systemic steroid burden that can be compared to previous results at the end of each quality improvement cycle. Participating sites should evaluate their change in process and outcomes as a result of the QIP, using the quality indicators and status reports.

\section{Further Program Evaluation}

Evaluation on the impact of the CONQUEST program will be conducted by OPRI in a series of cluster-randomized trials referred to as the "PRagmatic EVAluation of a quality Improvement program for people Living with modifiable high-risk COPD" (PREVAIL) trials (https://opri.org.uk/PREVAIL/). Currently, two separately powered PREVAIL trials are planned for the UK and the US, aiming to enroll a combined total of approximately 3000 patients from 250 sites over 18-36 months. The PREVAIL trials will compare patient outcomes including the rate of COPD exacerbations, rate of major adverse cardiovascular and respiratory events, and systemic corticosteroid usage between sites implementing the CONQUEST QIP and control sites continuing usual care. Results from the PREVAIL trials will be disseminated in a high-quality research journal to facilitate knowledge sharing on COPD QIPs.

\section{Conclusion}

The CONQUEST QIP represents a unique initiative to improve COPD care in patients with a modifiable but high risk of exacerbations, who stand to benefit most from timely diagnosis and management optimization. The core of the CONQUEST QIP lies in the implementation of a set of globally applicable, specifically designed, and expert-agreed quality standards into routine clinical practice. A global operational protocol outlining the implementation procedure will be provided to sites meeting the minimum healthcare system requirements who express interest in implementing the CONQUEST QIP.

Implementation of the CONQUEST QS is supported by CDS to assist in the prompt identification and diagnosis of patients with potential COPD, a crucial element in slowing disease progression and improving patient outcomes. ${ }^{62}$ The CONQUEST QIP requires routine collection and analysis of quality indicators and production of annual status reports 
informed by EMR, PRO, and PRI data. This guides and improves future quality improvement cycles, facilitating longterm implementation and improved patient outcomes via a cyclical process, incorporating feedback from previous cycles and sharing of knowledge within a learning network of healthcare professionals and the CONQUEST team. Continuous efforts are likely to be an important contributor to the success of the QIP. ${ }^{21}$

Ultimately, the goal of the CONQUEST QIP is to bolster the identification of modifiable high-risk COPD patients, embed and promote adherence to clinical guidelines in routine clinical practice, and optimize patient outcomes.

\section{Abbreviations}

CAT, COPD Assessment Test; CDS, clinical decision support; CONQUEST, COllaboratioN on QUality improvement initiative for achieving Excellence in STandards of COPD care; COPD, chronic obstructive pulmonary disease; EMR, electronic medical records; GOLD, Global Initiative for Chronic Obstructive Lung Disease; mMRC, modified Medical Research Council; OPC, Optimum Patient Care; OPRI, Observational and Pragmatic Research Institute; PREVAIL, PRagmatic EVAluation of a quality Improvement program for people Living with modifiable high-risk COPD; PRI, patient-reported information; PRO, patient-reported outcomes; QIP, quality improvement program; QS, quality standards.

\section{Acknowledgments}

Hana Muellerova, Patrick Darken, Paul Dorinsky, Frank Trudo, and Alex De Giorgio-Miller of AstraZeneca are acknowledged for their contribution to protocol development. Writing, editorial support, and/or formatting assistance in the development of this manuscript was provided by Shilpa Suresh, MSc, of the Observational and Pragmatic Research Institute, Singapore. Professor Dave Singh is supported by the National Institute for Health Research (NIHR) Manchester Biomedical Research Centre (BRC).

\section{Author Contributions}

The authors meet criteria for authorship as recommended by the International Committee of Medical Journal Editors. All authors made a significant contribution to the work reported, whether that is in the conception, study design, execution, acquisition of data, analysis, and interpretation, or in all these areas. All authors took part in drafting, revising or critically reviewing the article. All authors gave final approval of the version to be published. All authors have agreed on the journal to which the article has been submitted and agree to be accountable for all aspects of the work. All authors have given approval for the submission of this article. The authors received no direct compensation related to the development of the manuscript.

\section{Funding}

This study was conducted by Optimum Patient Care Global and the Observational and Pragmatic Research Institute (OPRI) Pte Ltd and was co-funded by Optimum Patient Care Global and AstraZeneca. No funding was received by the Observational \& Pragmatic Research Institute Pte Ltd (OPRI) for its contribution.

\section{Disclosure}

Luís Alves has served as an advisor or consultant for AstraZeneca, GlaxoSmithKline, and Merck Sharp \& Dohme; served as a speaker or a member of a speakers bureau for AstraZeneca, GlaxoSmithKline, BIAL, Viatris, and Novartis Pharmaceuticals Corporation. He is also a member of the Education Subcommittee of the International Primary Care Respiratory Group, member of the of GRESP, the Portuguese Primary Care Study Group for Respiratory Diseases. Rachel Pullen and Amy Couper are employees of the Observational and Pragmatic Research Institute, which is a research collaborator of the CONQUEST initiative with Optimum Patient Care and AstraZeneca. John Hurst has received personal payment and payment to his institution (UCL), including research grants, reimbursement for advisory work and educational activities, and support to attend meetings from pharmaceutical companies that make Medicines to treat COPD, which includes AstraZeneca, Boehringer Ingelheim, Chiesi and Novartis. Marc Miravitlles reports speaker fees from AstraZeneca, Boehringer Ingelheim, Chiesi, GlaxoSmithKline, Cipla, Menarini, Rovi, Bial, Sandoz, Zambon, CSL Behring, Grifols and Novartis, consulting fees from AstraZeneca, Atriva Therapeutics, 
Boehringer Ingelheim, Chiesi, GlaxoSmithKline, Bial, Gebro Pharma, Kamada, CSL Behring, Laboratorios Esteve, Ferrer, Mereo Biopharma, Verona Pharma, TEVA, Spin Therapeutics, Palobiofarma SL, pH Pharma, Novartis, ONO Pharma, Sanofi, Takeda, and Grifols and research grants from Grifols. Rongchang Chen has received grant support from AstraZeneca, Boehringer Ingelheim, and GSK; and reimbursement for advisory work and educational activities from AstraZeneca, Boehringer Ingelheim, GSK, Novartis. Mark Dransfield has received grant support from the Department of Defense and NIH; personal fees from AstraZeneca, Boehringer Ingelheim, PneumRx/BTG, Genentech, BostonScientific, Quark Pharmaceuticals and GSK; and contracted clinical trial support from Boehringer Ingelheim, Novartis, AstraZeneca, Yungjin, PneumRx/BTG, Pulmonx, Boston Scientific and GSK. Antony Hardjojo reports no conflict of interest. David Jones reports no conflict of interest. Rupert Jones declares grants from Astra Zeneca, Glaxo Smith Kline, Novartis and Teva and personal fees for consultancy, speakers fees or travel support from Astra Zeneca, Boehringer Ingelheim, Glaxo Smith Kline, Novartis, Nutricia, and OPRI. Victoria Carter, Alexander Evans, Margee Kerr and Marije van Melle are employees of Optimum Patient Care Ltd, a cofunder of the CONQUEST initiative. Marije van Melle reports grants from AstraZeneca, outside the submitted work. Konstantinos Kostikas was an employee and shareholder of Novartis Pharma AG until 31.10.2018. He has received honoraria for presentations and consultancy fees from AstraZeneca, Boehringer Ingelheim, Chiesi, CSL Behring, ELPEN, GSK, Menarini, Novartis, Sanofi Genzyme and WebMD. His Department has received funding and grants from AstraZeneca, Boehringer Ingelheim, Chiesi, Innovis, ELPEN, GSK, Menarini, Novartis and NuvoAir. He is a member of the GOLD Assembly. Jonathan Marshall is an employee of AstraZeneca and hold stock and/or stock options in the company. AstraZeneca is a co-funder of the CONQUEST initiative. Fernando Martinez has received personal fees and non-financial support from the American College of Chest Physicians, AstraZeneca, Bayer, Boehringer Ingelheim, CSL Behring, ConCert, Gala, Genentech, GSK, Inova Fairfax Health System, Miller Communications, National Society for Continuing Education, Novartis, Polarean, Pulmatrix, Pulmonx, Sanofi/ Regeneron, Pearl Pharmaceuticals, PeerView Communications, Prime Communications, Puerto Rico Respiratory Society, Chiesi, Sunovion, Theravance, Teva, Potomac, Verona, University of Alabama Birmingham, Physicians Education Resource, Canadian Respiratory Network, Teva and Dartmouth; non-financial support from ProterrixBio, Gilead, Nitto and Zambon; and personal fees from Columbia University, Integritas, MD magazine, Methodist Hospital Brooklyn, New York University, UpToDate, WebMD/MedScape, Western Connecticut Health Network, Patara/Respivant, PlatformIQ, American Thoracic Society, Rockpointe, Rare Disease Healthcare Communications and France Foundation; grant support from NIH; and is a member of steering committees for Afferent/Merck, Biogen, Veracyte, Prometic, Bayer, Bridge Biotherapeutics and ProMedior. Ruth Murray reports no conflicts of interest. Shigeo Muro reported lecture and advisory fees from Novartis Pharma, GlaxoSmithKline and AstraZeneca; lecture fees and grants from Boehringer Ingelheim; research grant from ROHTO Pharmaceutical and Kintetsu Cable Network, and grant and fund from Chugai Pharmaceutical, Ono Pharmaceutical, and Taiho Pharmaceutical. Clementine Nordon is an employee of AstraZeneca and hold stock and/or stock options in the company. AstraZeneca is a co-funder of the CONQUEST initiative. Michael Pollack is an employee of AstraZeneca and holds stock and/or stock options in the company. AstraZeneca is a co-funder of the CONQUEST initiative. Chris Price reports no conflicts of interest. Anita Sharma is a practicing Primary Care Physician and Senior Lecturer, School of Clinical Medicine-Primary Care Clinical Unit, University of Queensland. She supervises clinical training of primary care doctors and serves on advisory boards for Diabetes, Heart Failure and Osteoporosis for Novartis, Merck Sharp \& Dohme and Boehringer Ingelheim, Eli Lilley and Amgen. Dave Singh has received personal fees from Aerogen, AstraZeneca, Boehringer Ingelheim, Chiesi, Cipla, CSL Behring, Epiendo, Genentech, GlaxoSmithKline, Glenmark, Gossamerbio, Kinaset, Menarini, Novartis, Pulmatrix, Sanofi, Teva, Theravance and Verona. Tonya Winders has received personal fees from AstraZeneca, GSK, Novartis, Genentech, and Sanofi/ Regeneron alliance. Allergy \& Asthma Network \& Global Allergy \& Airways Patient Platform has received funds for unbranded disease awareness and education from AZ, BI, GSK, Novartis, Genentech, Viatris and Sanofi/ Regeneron. David B Price has advisory board membership with AstraZeneca, Boehringer Ingelheim, Chiesi, Mylan, Novartis, Regeneron Pharmaceuticals, Sanofi Genzyme, Thermofisher; consultancy agreements with 
Airway Vista Secretariat, AstraZeneca, Boehringer Ingelheim, Chiesi, EPG Communication Holdings Ltd, FIECON Ltd, Fieldwork International, GlaxoSmithKline, Mylan, Mundipharma, Novartis, OM Pharma SA, PeerVoice, Phadia AB, Spirosure Inc, Strategic North Limited, Synapse Research Management Partners S.L., Talos Health Solutions, Theravance and WebMD Global LLC; grants and unrestricted funding for investigator-initiated studies (conducted through Observational and Pragmatic Research Institute Pte Ltd) from AstraZeneca, Boehringer Ingelheim, British Lung Foundation, Chiesi, Merck, Mylan, Novartis, Regeneron Pharmaceuticals, Respiratory Effectiveness Group, Sanofi Genzyme, Theravance and UK National Health Service, Zentiva (Sanofi Generics); payment for lectures/ speaking engagements from AstraZeneca, Boehringer Ingelheim, Chiesi, Cipla, GlaxoSmithKline, Kyorin, Mylan, Mundipharma, Novartis, Regeneron Pharmaceuticals and Sanofi Genzyme; payment for travel/accommodation/meeting expenses from AstraZeneca, Boehringer Ingelheim, Mundipharma, Mylan, Novartis, Thermofisher; stock/stock options from AKL Research and Development Ltd which produces phytopharmaceuticals; owns $74 \%$ of the social enterprise Optimum Patient Care Ltd (Australia and UK) and 92.61\% of Observational and Pragmatic Research Institute Pte Ltd (Singapore); 5\% shareholding in Timestamp which develops adherence monitoring technology; is peer reviewer for grant committees of the UK Efficacy and Mechanism Evaluation programme, and Health Technology Assessment; and was an expert witness for GlaxoSmithKline. The authors report no other conflicts of interest in this work.

\section{References}

1. Global Initiative for Chronic Obstructive Lung Disease. Global Strategy for the Diagnosis, Management, and Prevention of Chronic Obstructive Pulmonary Disease; 2022. Available from: https://goldcopd.org/2022-gold-reports/. Accessed January 24, 2022.

2. World Health Organization. The top 10 causes of death. Available from: https://www.who.int/news-room/fact-sheets/detail/the-top-10-causes-ofdeath. Accessed April 20, 2021.

3. Morgan AD, Zakeri R, Quint JK. Defining the relationship between COPD and CVD: what are the implications for clinical practice? Ther Adv Respir Dis. 2018;12:1753465817750524.

4. Zafari Z, Li S, Eakin MN, Bellanger M, Reed RM. Projecting Long-Term Health and Economic Burden of COPD in the United States. Chest. 2020;159(4):1400.

5. Soriano JB, Kendrick PJ, Paulson KRet al. Prevalence and attributable health burden of chronic respiratory diseases, 1990-2017: a systematic analysis for the Global Burden of Disease Study 2017. Lancet Respir Med. 2020;8(6):585-596.

6. Halpin DMG, Hji DJ, Carter V, Skinner D, Price D. Distribution, Temporal Stability and Appropriateness of Therapy of Patients With COPD in the UK in Relation to GOLD 2019. EClinicalMedicine. 2019;14:32-41.

7. Kostikas K, Price D, Gutzwiller FS, et al. Clinical impact and healthcare resource utilization associated with early versus late COPD diagnosis in patients from UK CPRD Database. Int J Chron Obstruct Pulmon Dis. 2020;15:1729-1738.

8. Larsson K, Janson C, Ställberg B, et al. Impact of COPD diagnosis timing on clinical and economic outcomes: the ARCTIC observational cohort study. Int J Chron Obstruct Pulmon Dis. 2019;14:995-1008.

9. Crisan L, Wong N, Sin DD, Lee HM. Karma of cardiovascular disease risk factors for prevention and management of major cardiovascular events in the context of acute exacerbations of chronic obstructive pulmonary disease. Front Cardiovasc Medi. 2019;6:79.

10. Kunisaki KM, Dransfield MT, Anderson JA, et al. Exacerbations of chronic obstructive pulmonary disease and cardiac events. a post hoc cohort analysis from the SUMMIT randomized clinical trial. Am J Respir Crit Care Med. 2018;198(1):51-57. doi:10.1164/rccm.201711$22390 \mathrm{OC}$

11. Donaldson GC, Seemungal TAR, Bhowmik A, Wedzicha JA. Relationship between exacerbation frequency and lung function decline in chronic obstructive pulmonary disease. Thorax. 2002;57(10):847.

12. Kerkhof M, Voorham J, Dorinsky P, et al. The long-term burden of COPD exacerbations during maintenance therapy and lung function decline. Int J Chron Obstruct Pulmon Dis. 2020;15:1909-1918.

13. Seemungal TAR, Donaldson GC, Paul EA, Bestall JC, Jeffries DJ, Wedzicha JA. Effect of exacerbation on quality of life in patients with chronic obstructive pulmonary disease. Am J Respir Crit Care Med. 1998;157(5):1418-1422.

14. Soler-Cataluña JJ, Martínez-García MA, Román Sánchez P, Salcedo E, Navarro M, Ochando R. Severe acute exacerbations and mortality in patients with chronic obstructive pulmonary disease. Thorax. 2005;60(11):925-931.

15. Suissa S, Dell AS, Ernst P. Long-term natural history of chronic obstructive pulmonary disease: severe exacerbations and mortality. Thorax. 2012;67(11):957.

16. Jones RCM, Price D, Ryan D, et al. Opportunities to diagnose chronic obstructive pulmonary disease in routine care in the UK: a retrospective study of a clinical cohort. Lancet Respir Med. 2014;2(4):267-276.

17. Health Resources and Services Administration. Quality Improvement. April, 2011. Available from:https://www.hrsa.gov/sites/default/files/quality/ toolbox/508pdfs/qualityimprovement.pdf. Accessed:April 20, 2021.

18. American Academy of Family Physicians. Basics of Quality Improvement. Available from: https://www.aafp.org/family-physician/practice-andcareer/managing-your-practice/quality-improvement-basics.html. Accessed April 20, 2021.

19. Mehanni S, Jha D, Kumar A, et al. Implementing a quality improvement initiative for the management of chronic obstructive pulmonary disease in rural Nepal. BMJ Open Qual. 2019;8(1):e00408.

20. Morganroth M, Pape G, Rozenfeld Y, Heffner JE. Multidisciplinary COPD disease management program: impact on clinical outcomes. Postgrad Med. 2016;128(2):239-249. 
21. Hurst JR, Quint JK, Stone RA, Silove Y, Youde J, Roberts CM. National clinical audit for hospitalised exacerbations of COPD. ERJ Open Res. 2020;6:3.

22. Terasaki J, Singh G, Zhang W, Wagner P, Sharma G. Using EMR to improve compliance with clinical practice guidelines for management of stable COPD. Respir Med. 2015;109(11):1423-1429.

23. Martinez FJ, Thomashow B, Sapir T, Simone L, Carter J, Han M. Does evaluation and management of COPD follow therapeutic strategy recommendations? Chronic Obstructive Pul Dis. 2021;8(2):230-242.

24. Trout D, Bhansali AH, Riley DD, Peyerl FW, Lee-Chiong TL Jr. A quality improvement initiative for COPD patients: a cost analysis. PLoS One. 2020;15(7):e0235040.

25. Burkes RM, Mkorombindo T, Chaddha U, et al. Impact of quality improvement on care of chronic obstructive pulmonary disease patients in an internal medicine resident clinic. Healthcare (Basel). 2018;6(3):88.

26. Ferrone M, Masciantonio MG, Malus N, et al. The impact of integrated disease management in high-risk COPD patients in primary care. npj Prim Care Respir Med. 2019;29(1):1-9. doi:10.1038/s41533-019-0119-9

27. Cosío BG, Shafiek H, Verdú J, et al. Implementation of an integrated care model for frequent-exacerbator COPD patients: a controlled prospective study. Arch Bronconeumol. 2021;57(9):577-583. doi:10.1016/j.arbres.2021.01.025

28. Poot CC, Meijer E, Kruis AL, Smidt N, Chavannes NH, Honkoop PJ. Integrated disease management interventions for patients with chronic obstructive pulmonary disease. Cochrane Database Syst Rev. 2021;(9). doi:10.1002/14651858.CD009437.pub3

29. Wu CX, Hwang CH, Tan WS, et al. Effectiveness of a chronic obstructive pulmonary disease integrated care pathway in a regional health system: a propensity score matched cohort study. BMJ Open. 2018;8(3):e019425. doi:10.1136/bmjopen-2017-019425

30. Gaveikaite V, Grundstrom C, Winter S, Chouvarda I, Maglaveras N, Priori R. A systematic map and in-depth review of European telehealth interventions efficacy for chronic obstructive pulmonary disease. Respir Med. 2019;158:78-88. doi:10.1016/j.rmed.2019.09.005

31. Lenferink A, Brusse-Keizer M, van der Valk PD, et al. Self-management interventions including action plans for exacerbations versus usual care in patients with chronic obstructive pulmonary disease. Cochrane Database Syst Rev. 2017;8:CD011682. doi:10.1002/14651858. CD011682.pub2

32. Kruis AL, Boland MRS, Assendelft WJJ, et al. Effectiveness of integrated disease management for primary care chronic obstructive pulmonary disease patients: results of cluster randomised trial. BMJ. 2014;349(sep1011):g5392-g5392. doi:10.1136/bmj.g5392

33. Lanning E, Longstaff J, Jones T, et al. Modern Innovative Solutions in Improving Outcomes in Chronic Obstructive Pulmonary Disease (MISSION COPD): mixed methods evaluation of a novel integrated care clinic. Interact J Med Res. 2019;8(4):e9637. doi:10.2196/ijmr.9637

34. Yawn BP, Duvall K, Peabody J, et al. The impact of screening tools on diagnosis of chronic obstructive pulmonary disease in primary care. Am J Prev Med. 2014;47(5):563-575.

35. Jordan RE, Adab P, Sitch A, et al. Targeted case finding for chronic obstructive pulmonary disease versus routine practice in primary care (TargetCOPD): a cluster-randomised controlled trial. Lancet Respir Med. 2016;4(9):720-730.

36. Jones $\mathrm{P}$, Miravitlles $\mathrm{M}$, Molen $\mathrm{T}$, et al. Beyond $\mathrm{FEV}_{1}$ in $\mathrm{COPD}$ : a review of patient-reported outcomes and their measurement. COPD. 2012;7:697-709. doi:10.2147/COPD.S32675

37. Moreo K, Greene L, Sapir T. Improving interprofessional and coproductive outcomes of care for patients with chronic obstructive pulmonary disease. BMJ Open Qual. 2016;5(1):u210329.w4679. doi:10.1136/bmjquality.u210329.w4679

38. Evans A, Soremekun S, Stanley B, et al. Strategies that promote sustainability in quality improvement activities for chronic disease management in healthcare settings: a Practical Perspective. Qual Prim Care. 2020;28(6):55.

39. Pace WD, Brandt E, Carter VA, et al. Characterization of COPD in U.S. primary care: data from the optimum patient care with DARTNet research database and the embedded Advancing the Patient Experience (APEX) in COPD registry. Ann Fam Med. 2022 Jan 7.

40. Ryan D, Ryan F, Mascarenhas L, et al. P276 The impact of the optimum patient care service on outcomes for adult asthmatic patients. Thorax. 2012;67(Suppl 2):A186.

41. Ray E, Culliford D, Kruk H, et al. Specialist respiratory outreach: a case-finding initiative for identifying undiagnosed COPD in primary care. NPJ Prim Care Respir Med. 2021;31(1):7.

42. Magnussen H, Lucas S, Lapperre T, et al. Withdrawal of inhaled corticosteroids versus continuation of triple therapy in patients with COPD in real life: observational comparative effectiveness study. Respir Res. 2021;22(1):25.

43. Kerkhof M, Voorham J, Dorinsky P, et al. Association between COPD exacerbations and lung function decline during maintenance therapy. Thorax. 2020;75(9):744-753.

44. Kostikas K, Rhee CK, Hurst JR, et al. Adequacy of therapy for people with both COPD and heart failure in the UK: historical cohort study. Prag Observational Re. 2020;11:55-66.

45. Yawn BP, Kaplan A, Pace WD, et al. Advancing the Patient EXperience (APEX) in COPD registry: study design and strengths. $J$ Am Board Fam Med. 2021;34(1):22-31.

46. Edwards CL, Kaplan AG, Yawn BP, et al. Development of the Advancing the Patient Experience in COPD registry: a modified delphi study. Chronic Obstructive Pul Dis. 2021;8:1.

47. Pullen R, Miravitlles M, Sharma A, et al. CONQUEST quality standards: for the collaboration on quality improvement initiative for achieving excellence in standards of COPD care. Int J Chron Obstruct Pulmon Dis. 2021;16:2301-2322.

48. Advancing Care Coordination and Telehealth (CC\&TH) deployment at Scale. Kronikgune, Basque Country. ACT@Scale Content; 2021. Available from: https://www.kronikgune.org/en/research-projects/european-projects-and-initiatives/others-european-projects/act-scale/. Accesssed December $15,2021$.

49. Schonenberg H, Nielsen E, Syse T, Bescos C. Experiences on Scaling Care Coordination and Telehealth Best Practice. Koninklijke Philips N.V.; 2019. Available from: https:/www.philips.com/c-dam/corporate/newscenter/global/standard/resources/healthcare/2019/act-at-scale/ACT-atScale_Handbook.download.pdf. Accessed December 15, 2021.

50. Royal College of General Practitioners. Quality Improvement for General Practice. A Guide for GPs and the Whole Practice Team. Royal College of General Practitioners; 2015. Available from: https:/www.rcgp.org.uk/-/media/Files/CIRC/Quality-Improvement/RGCP-QI-Guide-260216.ashx? la=en. Accessed December 15, 2021.

51. Higgins TC, Crosson J, Peikes D, McNellis R, Genevro J, Meyers D. Using Health Information Technology to Support Quality Improvement in Primary Care. AHRQ Publication No. 15-0031- EF. Rockville, MD: Agency for Healthcare Research and Quality; 2015. 
52. Hurst JR, Winders T, Worth H, et al. A patient charter for chronic obstructive pulmonary disease. Adv Ther. 2021;38(1):11-23.

53. Halpin DM, Decramer M, Celli B, Kesten S, Leimer I, Tashkin DP. Risk of nonlower respiratory serious adverse events following COPD exacerbations in the 4-year UPLIFT ${ }^{\circledR}$ trial. Lung. 2011;189(4):261-268.

54. Rothnie KJ, Müllerová H, Hurst JR, et al. Validation of the recording of acute exacerbations of COPD in UK primary care electronic healthcare records. PLoS One. 2016;11(3):e0151357.

55. Health Data Research UK. Welcome to CALIBER! Available from: https://www.caliberresearch.org/portal. Accessed April 20, 2020.

56. José Soler-Cataluña J, Alcázar-Navarrete B, Miravitlles M. The concept of control in COPD: a new proposal for optimising therapy. Eur Respir J. 2014;44(4):1072.

57. Miravitlles M, Sliwinski P, Rhee CK, et al. Changes in control status of COPD over time and their consequences: a prospective international study. Arch Bronconeumol (Engl Ed). 2021;57(2):122-129.

58. Soler-Cataluña JJ, Marzo M, Catalán P, Miralles C, Alcazar B, Miravitlles M. Validation of clinical control in COPD as a new tool for optimizing treatment. Int J Chron Obstruct Pulmon Dis. 2018;13:3719-3731.

59. New Advances in Collecting and Using Patient-Reported Outcome Data. Agency for Healthcare Research and Quality. Rockville, MD.; 2020. Available from: https://www.ahrq.gov/news/blog/ahrqviews/prism-app.html. Accessed August 25, 2021.

60. Weldring T, Smith SMS. Article Commentary: patient-Reported Outcomes (PROs) and Patient-Reported Outcome Measures (PROMs). Health ServInsights. 2013;6:S11093.

61. Kocks JWH, Seys SF, van Duin TS, Diamant Z, Tsiligianni IG. Assessing patient-reported outcomes in asthma and COPD patients: which can be recommended in clinical practice? Curr Opin Pulm Med. 2018;24(1):18-23.

62. Welte T, Vogelmeier C, Papi A. COPD: early diagnosis and treatment to slow disease progression. Int J Clin Pract. 2015;69(3):336-349.

63. Martinez FJ, Mannino D, Leidy NK, et al. A new approach for identifying patients with undiagnosed chronic obstructive pulmonary disease. Am J Respir Crit Care Med. 2017;195(6):748-756.

64. Price DB, Tinkelman DG, Nordyke RJ, Isonaka S, Halbert RJ. Scoring system and clinical application of COPD diagnostic questionnaires. Chest. 2006;129(6):1531-1539.

Patient Related Outcome Measures

\section{Dovepress}

\section{Publish your work in this journal}

Patient Related Outcome Measures is an international, peer-reviewed, open access journal focusing on treatment outcomes specifically relevant to patients. All aspects of patient care are addressed within the journal and practitioners from all disciplines are invited to submit their work as well as healthcare researchers and patient support groups. The manuscript management system is completely online and includes a very quick and fair peer-review system. Visit http://www.dovepress.com/testimonials.php to read real quotes from published authors.

Submit your manuscript here: http://www.dovepress.com/patient-related-outcome-measures-journal 